\title{
DONNER VoiX AUX MUltiples REPRÉSENTATIONS DU DÉVELOPPEMENT DURABLE DANS LES FORMATIONS EN GÉNIE
}

\author{
Olivier Riffon et Claude Villeneuve \\ Chaire en éco-conseil, Université du Québec à Chicoutimi \\ olivier_riffon@uqac.ca, claude_villeneuve@uqac.ca
}

\begin{abstract}
Résumé : Le développement durable (DD) est un concept polysémique, pouvant être interprété différemment selon les acteurs et les organisations qui se l'approprient. Les ingénieurs abordent souvent les problématiques et projets identifiés au DD sous un angle technique et économique. Dans l'exercice de leur profession, ils sont toutefois confrontés à des interprétations diverses $d u \mathrm{DD}$, portées par des parties prenantes aux intérêts variés et quelquefois divergents. Pour faciliter la communication et la compréhension mutuelle, la Chaire en éco-conseil de l'UQAC a développé une typologie des interprétations $d u$ $D D$. Cette typologie a été utilisée à deux reprises dans des formations offertes à des ingénieurs, appuyée par l'analyse d'un projet fictif au moyen d'une grille de DD. L'utilisation de ces outils dans la formation des ingénieurs vise à mieux les préparer pour le travail au sein d'équipe multidisciplinaire et à communiquer plus efficacement avec le public sur des projets de DD.
\end{abstract}

Mots clés : Développement durable, formation, typologie, grille d'analyse, génie, éco-conseil.

\section{INTRODUCTION}

Depuis son entrée dans le vocabulaire, le développement durable (DD) est devenu un concept faisant l'objet d'un large consensus social [12] sur son objectif, tant auprès des organisations économiques, sociocommunautaires, environnementales et politiques. Le concept, d'abord proposé et promu lors de grandes rencontres internationales tenues sous l'égide de l'ONU, a évolué avec la pratique dans les divers secteurs de l'activité humaine. En touchant plusieurs types d'acteurs et d'organisations, il a nécessairement pris différents visages. En conséquence, l'interprétation de la définition du DD tend à varier selon les besoins, la culture et la hiérarchie des valeurs de celui qui s'en approprie l'intention. De même, les outils utilisés pour la mise en œuvre du DD varient selon le contexte et les besoins [8].

Les ingénieurs, par la nature de leur formation, de leurs compétences et de leurs pratiques, abordent souvent les problématiques et projets identifiés à une intention de DD sous un angle technique et économique. Dans l'exercice de leur profession, ils sont toutefois confrontés à des interprétations diverses du DD, portées par des parties prenantes aux intérêts variés et parfois divergents. C'est le cas dans les équipes multidisciplinaires en charge de la gestion de projets d'ingénierie, et encore plus lors d'exercices d'examen public des projets. Différentes interprétations peuvent également être portées par les firmes qui les engagent, par leurs clients, par les groupes d'influence autour des projets, etc.

Pour faciliter la communication et la compréhension mutuelle, une typologie des interprétations du DD peut être utile. Des chercheurs de la Chaire en éco-conseil de I'UQAC ont développé une typologie des conceptions, finalités, approches et stratégies du DD [8], afin d'aider les professionnels à identifier la compréhension qu'ont les parties prenantes d'un projet et à leur proposer des stratégies et des outils appropriés [9].

Afin de familiariser les ingénieurs à d'autres interprétations du DD qui coexistent dans la société, cette typologie a été utilisée à deux reprises dans des formations offertes à des ingénieurs, appuyée par l'utilisation d'une grille d'analyse de projets développée par la Chaire en éco-conseil [13]. Les participants ont utilisé ces outils dans un exercice pratique sur un cas fictif de projet d'ingénierie. La communication proposée vise à présenter la typologie et la grille d'analyse développées, ainsi qu'à démontrer comment l'utilisation de ces outils dans la formation des ingénieurs les prépare pour le travail en équipe multidisciplinaire et pour la communication avec divers publics sur des projets de DD.

\section{TYPOLOGIE D'UN CONCEPT}

La littérature en matière de développement durable est riche, et elle comporte entre autre une multitude de propositions de typologie [1]. La structure de la typologie développée par la chaire en éco-conseil fait place à la complexité en se déclinant en quatre niveaux, présentant des caractéristiques distinctes et complémentaires du

CEEA13;

Montreal, QC; June 17-20, 2013 
concept, de façon à définir une représentation complète du $\mathrm{DD}$, tel que présenté à la figure 1 .

- La conception du DD : fondement de la compréhension du développement durable;

- La finalité du DD : objectifs motivants l'adhésion au concept;

- L'approche du DD : philosophie de l'action ;

- Les stratégies de mise en œuvre du DD : méthodes et outils mobilisés pour l'application.

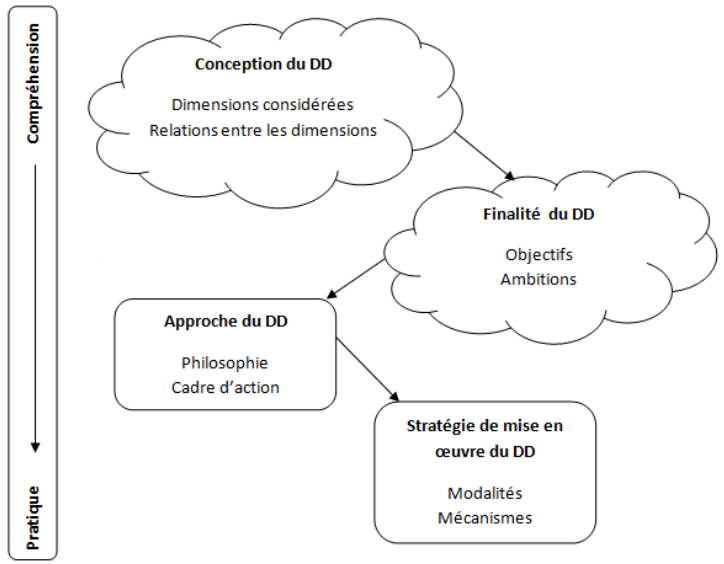

Figure 1. Les 4 niveaux typologiques

\subsection{Conception du DD}

Une conception est définie comme la façon de voir, d'élaborer dans son esprit un ensemble de choses complexes. La conception représente le fondement de la compréhension et de la vision du DD d'un acteur. C'est une construction abstraite qui peut être illustrée par un modèle statique ou dynamique. Elle se définit par le nombre et la nature des dimensions considérées (sociale, écologique, économique, éthique, culturelle, territoriale, de gouvernance, etc.) ainsi que par les relations entre ces dimensions (égalité, priorité, hiérarchie, subordination). Quelques exemples sont illustrés dans les figures 2 à 5 , où sont entre autres présentés les modèles de Jacob et Sadler [4], de Passet [6] et de Revéret et Gendron [7].

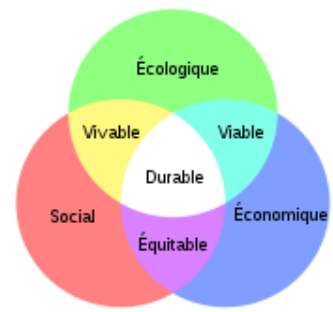

Figure 2. Modèle de Jacob et Sadler : Conception à 3 dimensions égalitaires

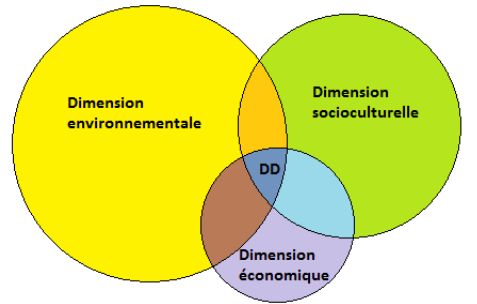

Figure 3. Conception à trois dimensions priorisées

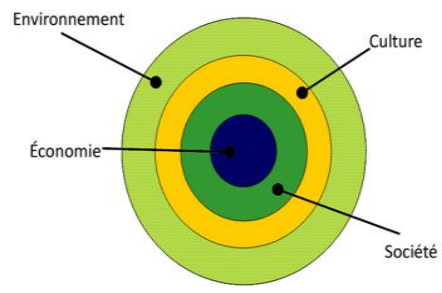

Figure 4. Modèle de Passet : Conception à 4 dimensions subordonnées

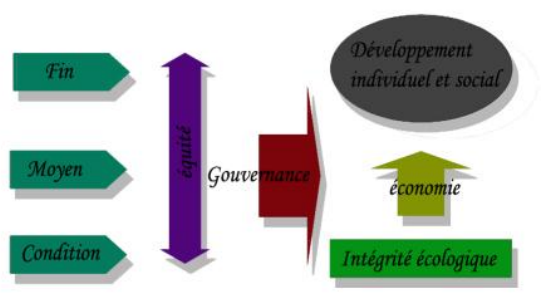

Figure 5. Modèle de Revéret et Gendron : Conception à 5 dimensions hiérarchisées

\subsection{Finalité du DD}

La finalité définit ce qui se trouve au cœur des enjeux et des objectifs du DD, ce qui justifie que des efforts soient investis ou des changements soient apportés. Elle se construit à partir de valeurs et d'intérêts et nous renseigne sur la hiérarchie (consciente ou inconsciente) qu'un acteur établit entre les dimensions du développement durable [10]. Les finalités du DD sont résumées dans le tableau 1.

Tableau 1. Résumé des finalités du DD

\begin{tabular}{|l|l|}
\hline Biocentrée & $\begin{array}{l}\text { Axée sur la nature, sur le maintien ou la } \\
\text { protection du patrimoine naturel contre } \\
\text { l'intervention humaine }\end{array}$ \\
\hline Écocentrée & $\begin{array}{l}\text { Axée sur la préservation des ressources, } \\
\text { l'humain faisant partie des écosystèmes. }\end{array}$ \\
\hline Anthropocentrée & Axée sur le bien-être des individus \\
\hline Sociocentrée & $\begin{array}{l}\text { Axé sur le bien-être des individus dans } \\
\text { leur structure sociale }\end{array}$ \\
\hline Technocentrée & $\begin{array}{l}\text { Axée sur le progrès scientifique et } \\
\text { technique }\end{array}$ \\
\hline Multicentrée & $\begin{array}{l}\text { Axée sur l'évolution de l'humain devant } \\
\text { développer sa conscience et sa } \\
\text { responsabilité vis-à-vis de la nature }\end{array}$ \\
\hline
\end{tabular}

CEEA13; 


\subsection{Approche du DD}

L'approche est une philosophie d'action qui teinte les modalités de mise en œuvre du DD à l'échelle de l'organisation, en lien avec les représentations dominantes et le contexte organisationnel. Elle se défini en fonction des grandes orientations préconisées pour le développement [3]. Les principales approches du DD sont résumées dans le tableau 2.

Tableau 2. Résumé des approches du DD

\begin{tabular}{|c|c|}
\hline Approche & Objectifs et concepts clés \\
\hline Économiciste & $\begin{array}{l}\text { Vise la croissance économique et la } \\
\text { satisfaction des besoins humains. } \\
\text { L'environnement et les responsabilités } \\
\text { sociales sont instrumentalisés par des outils } \\
\text { du marché. }\end{array}$ \\
\hline $\begin{array}{l}\text { Environne- } \\
\text { mentaliste }\end{array}$ & $\begin{array}{l}\text { Vise l'intégrité des écosystèmes, de la } \\
\text { biosphère et de la biodiversité. Le capital } \\
\text { naturel est considéré comme inaliénable. }\end{array}$ \\
\hline $\begin{array}{l}\text { Écologiste ou } \\
\text { systémique }\end{array}$ & $\begin{array}{l}\text { Vise la recherche de l'équilibre entre les } \\
\text { dimensions du développement, dans une } \\
\text { approche systémique et intégrée. } \\
\text { L'environnement, la société et l'économie } \\
\text { sont perçus comme interdépendants. }\end{array}$ \\
\hline Humaniste & $\begin{array}{l}\text { Vise le bien-être de l'humain et la qualité } \\
\text { de vie. Les rapports équitables, la justice } \\
\text { sociale, la solidarité, la diversité culturelle, } \\
\text { le renforcement des capacités sont au cœur } \\
\text { de cette approche. }\end{array}$ \\
\hline Territoriale & $\begin{array}{l}\text { Vise l'application locale du DD pour en } \\
\text { augmenter les chances de succès. Cette } \\
\text { approche améliore la prise en compte des } \\
\text { inégalités sociales et écologiques, en } \\
\text { valorisant la gouvernance territoriale. }\end{array}$ \\
\hline Politique & $\begin{array}{l}\text { Vise l'intégration du développement } \\
\text { durable dans les processus de prise de } \\
\text { décision. Ce doit être fait avec une vision } \\
\text { élargie qui comprend l'atteinte de buts } \\
\text { écologiques, sociaux et économiques, } \\
\text { déterminés collectivement }\end{array}$ \\
\hline Scientifique & $\begin{array}{l}\text { Vise l'élaboration et la diffusion des } \\
\text { connaissances, l'analyse des dynamiques } \\
\text { humaines et naturelles, l'innovation } \\
\text { technologique et technique. La science et la } \\
\text { technologie peuvent régler les problèmes } \\
\text { de développement durable. }\end{array}$ \\
\hline Éthique & $\begin{array}{l}\text { Vise l'identification des valeurs communes } \\
\text { et la cohérence entre ces valeurs et les } \\
\text { actions. Se base sur un questionnement } \\
\text { continuel des discours et des actions. }\end{array}$ \\
\hline
\end{tabular}

\subsection{Stratégie d'application du DD}

Les stratégies sont associées à l'action et aux modalités de mise en cuvre du DD. Elles nous renseignent sur la nature des outils utilisés, les modalités de planification et de mise en œuvre, l'autorité compétente pour juger de la validité de la démarche, l'ampleur des changements attendus, etc. Il existe de nombreuses typologies des réponses stratégiques des organisations en matière de DD [5]. Notre typologie propose douze caractéristiques stratégiques d'une démarche de $\mathrm{DD}$, présentées sous forme dichotomique, où les termes marquent des extrêmes entre lesquels se situe la stratégie réelle d'une organisation. Ces 24 stratégies sont résumées dans le tableau 3.

Tableau 3. Résumé des stratégies du DD

\begin{tabular}{|c|c|}
\hline \multicolumn{2}{|c|}{ Direction } \\
\hline $\begin{array}{l}\text { Descendante : le sommet } \\
\text { décisionnel identifie les } \\
\text { problématiques et coordonne } \\
\text { les actions locales ou } \\
\text { sectorielles }\end{array}$ & $\begin{array}{l}\text { Ascendante : les gens sur le } \\
\text { terrain décident des } \\
\text { objectifs prioritaires et des } \\
\text { façons de mettre en œuvre } \\
\text { le développement durable }\end{array}$ \\
\hline \multicolumn{2}{|c|}{$\begin{array}{ll}\text { Cadre } \\
\end{array}$} \\
\hline $\begin{array}{l}\text { Prescriptif : une organisation } \\
\text { externe sert de référence, fixe } \\
\text { les objectifs et exige une } \\
\text { reddition de comptes }\end{array}$ & $\begin{array}{l}\text { Volontaire : la démarche est } \\
\text { conçue à l'interne, } \\
\text { l'organisation ne rend des } \\
\text { comptes qu'à elle-même }\end{array}$ \\
\hline \multicolumn{2}{|c|}{ Participation } \\
\hline $\begin{array}{l}\text { Participative : favorise } \\
\text { l'implication active des } \\
\text { parties prenantes aux } \\
\text { processus décisionnels }\end{array}$ & $\begin{array}{l}\text { Autoritaire : les objectifs, } \\
\text { méthodes et priorités sont } \\
\text { imposés par un secteur ou } \\
\text { un responsable, avec peu de } \\
\text { participation }\end{array}$ \\
\hline \multicolumn{2}{|c|}{ Gestion } \\
\hline $\begin{array}{l}\text { Pragmatique : le DD } \\
\text { progresse par cumul de } \\
\text { projets et d'initiatives, selon } \\
\text { les urgences ou opportunités }\end{array}$ & $\begin{array}{l}\text { Planificatrice : l'utilisation } \\
\text { d'outils de planification } \\
\text { favorise une démarche } \\
\text { d'amélioration continue }\end{array}$ \\
\hline \multicolumn{2}{|c|}{ Portée } \\
\hline $\begin{array}{l}\text { Globale : le DD est appliqué à } \\
\text { large échelle, dans l'ensemble } \\
\text { des secteurs ou des territoires } \\
\text { d'une organisation }\end{array}$ & $\begin{array}{l}\text { Sectorielle : le DD est } \\
\text { appliqué à l'échelle locale, } \\
\text { dans un secteur ou un } \\
\text { territoire spécifique }\end{array}$ \\
\hline \multicolumn{2}{|c|}{ Dépendance } \\
\hline $\begin{array}{l}\text { Endogène : la démarche est } \\
\text { menée à partir des ressources } \\
\text { interne (à l'organisation ou au } \\
\text { territoire), de façon } \\
\text { indépendante }\end{array}$ & $\begin{array}{l}\text { Exogène : la démarche est } \\
\text { dépendante des ressources } \\
\text { externe (à l'organisation ou } \\
\text { au territoire), elle mobilise } \\
\text { peu de ressources internes }\end{array}$ \\
\hline \multicolumn{2}{|c|}{ Prospective } \\
\hline $\begin{array}{l}\text { Prévisionnelle (forecast): } \\
\text { l'analyse des tendances } \\
\text { permet l'élaboration des } \\
\text { scénarios futurs pour guider } \\
\text { les actions }\end{array}$ & $\begin{array}{l}\text { Rétrospective (backcast) : la } \\
\text { vision du futur souhaité } \\
\text { mène à l'élaboration } \\
\text { d'actions pour atteindre } \\
\text { cette vision }\end{array}$ \\
\hline \multicolumn{2}{|c|}{ Intégration } \\
\hline $\begin{array}{l}\text { Parallèle : le DD est abordé } \\
\text { par une démarche parallèle, } \\
\text { complémentaire aux activités } \\
\text { de gestion de l'organisation }\end{array}$ & $\begin{array}{l}\text { Intégrée : les principes de } \\
\text { DD sont systématiquement } \\
\text { intégrés dans les actions et } \\
\text { outils de l'organisation }\end{array}$ \\
\hline \multicolumn{2}{|c|}{ Initiative } \\
\hline $\begin{array}{l}\text { Réactive : la démarche est } \\
\text { entreprise en réaction à une } \\
\text { situation critique, à une } \\
\text { menace, à une obligation }\end{array}$ & $\begin{array}{l}\text { Proactive ; la démarche est } \\
\text { entreprise sans pression } \\
\text { (interne ou externe), faisant } \\
\text { preuve de leadership }\end{array}$ \\
\hline
\end{tabular}

CEEA13; 


\begin{tabular}{|l|l|}
\hline \multicolumn{2}{|c|}{ Adaptation } \\
\hline $\begin{array}{l}\text { Réformatrice : la démarche } \\
\text { progresse de façon évolutive, } \\
\text { par le principe de } \\
\text { l'amélioration continue }\end{array}$ & $\begin{array}{l}\text { Transformatrice : la } \\
\text { démarche représente une } \\
\text { rupture qui modifie en } \\
\text { profondeur la gestion } \\
\text { organisationnelle }\end{array}$ \\
\hline \multicolumn{2}{|c|}{ Orientation } \\
\hline $\begin{array}{l}\text { Vers l'interne : les impacts } \\
\text { projetés de la démarche sont } \\
\text { davantage orientés vers les } \\
\text { publics internes }\end{array}$ & $\begin{array}{l}\text { Vers l'externe : les impacts } \\
\text { projetés de la démarche sont } \\
\text { davantage orientés vers les } \\
\text { publics externes }\end{array}$ \\
\hline \multicolumn{2}{|c|}{ Contrainte } \\
\hline $\begin{array}{l}\text { Sous contrainte : la démarche } \\
\text { est soumise à plusieurs } \\
\text { contraintes internes et } \\
\text { externes, limitant la portée et } \\
\text { le rythme d'implantation }\end{array}$ & $\begin{array}{l}\text { Sans contrainte : les } \\
\text { contraintes (interne ou } \\
\text { externe) sont minimes et } \\
\text { l'organisation dispose de } \\
\text { marges de manœuvre }\end{array}$ \\
\hline
\end{tabular}

Les quatre niveaux typologiques définis sont complémentaires, allant d'un intérêt plus conceptuel à un angle plus appliqué. Certains liens peuvent évidemment être faits entre les conceptions, les finalités, les approches et les stratégies d'application. Certaines combinaisons peuvent être facilement repérées: il est entre autre possible de lier certaines approches avec des finalités dominantes. Par exemple, lorsqu'il est question de DD dans les formations en génie, la combinaison entre la finalité technocentrée et l'approche technoscientifique ou économiciste est très présente.

Notons également que les frontières entre les niveaux typologiques ne sont pas toujours claires et franches. Certains termes se superposent, se recoupent, s'hybrident. Comme la plupart des typologies du DD, elle superpose une classification artificielle, sommaire et simplifiée, nécessairement limitée en contenu (Dobson, 1996). La nature complexe et polysémique du DD fait qu'il est difficile d'y plaquer des étiquettes. L'ensemble des interprétations permet de constater que le DD reste toujours lié à la complexité, ce qui peut être confrontant pour les ingénieurs, qui sont souvent formés à résoudre des problèmes en les réduisant à leurs composantes.

\section{GRILLE D'ANALYSE DU DÉVELOPPEMENT DURABLE}

La typologie a été utilisée à deux reprises dans des formations offertes à des ingénieurs par des membres de la chaire en éco-conseil, afin de présenter des éléments qui sortent de leur interprétation classique. Pour rendre ces apprentissages concrets, la typologie est appuyée par l'utilisation d'une grille d'analyse développée par la Chaire en éco-conseil [13] et appliquée à de nombreux projets, dont le projet de la Mine Arnaud à Sept-Îles [11].

La grille d'analyse de DD de la Chaire en éco-conseil est un outil de questionnement selon cinq dimensions (écologique, sociale, économique, éthique, de gouvernance, voir la figure 6) qui permet d'évaluer dans quelle mesure un projet ou une démarche d'une organisation favorise l'amélioration des conditions humaines par son action sur le réel. Elle permet à ceux qui l'appliquent de se situer et de proposer des pistes de bonification à un projet ou à une démarche, dans une perspective d'amélioration continue. L'analyse peut aussi servir à fixer des objectifs, à identifier des indicateurs, à éclairer la prise de décision ou à trouver des compromis favorisant l'acceptabilité sociale d'un projet [14].

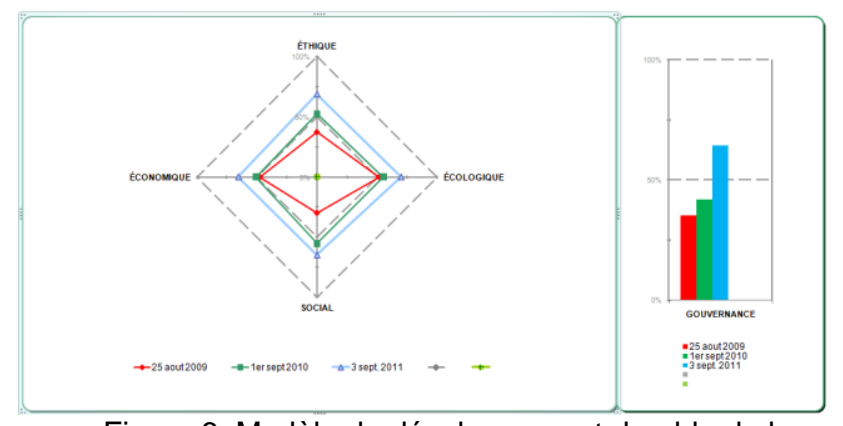

Figure 6. Modèle de développement durable de la Chaire en éco-conseil

Cette grille d'analyse est constituée de principes, de thèmes et d'objectifs inspirés de l'analyse des textes des grandes conférences et conventions internationales (Stratégie mondiale de la Conservation, Commission Brundtland, Stratégie pour l'avenir de la Vie, Agenda 21, Objectifs du millénaire, etc.). Les objectifs proposés pour chaque dimension servent à composer un indice qualitatif de la capacité d'un projet à satisfaire aux conditions du DD. Cette démarche a l'avantage de rejoindre à la fois les préoccupations globales et les évaluations d'impacts de type plus local, limitées dans l'espace et dans le temps.

La grille est composée de cinq tableaux. Chaque tableau propose un principe et des thèmes. Ces thèmes se déclinent en objectifs qui peuvent être pondérés et évalués en fonction de l'application (action, projet, démarche, etc.). Chaque dimension considérée correspond à ces principes spécifiques et vise à répondre à cinq types de besoins précis [14].

\section{RESULTATS D'UN EXERCICE CONCRET POUR DES INGENIEURS EN FORMATION}

Dans les deux interventions réalisées auprès des ingénieurs en formation, les étudiants ont utilisé ces deux outils, la typologie et la grille d'analyse, dans un exercice pratique réalisé sur un cas fictif de projet d'ingénierie.

Le cas fictif proposé est celui de la construction d'une voie de contournement d'un village situé à proximité d'un grand centre. Le cas présente une carte avec le trajet proposé, ainsi qu'un texte descriptif du contexte ayant mené à l'élaboration de ce projet. Les principales caractéristiques techniques du projet sont précisées, ainsi que les enjeux, problématiques et contestations sociales,

CEEA13; 
environnementales et économiques que le projet soulève. Les étudiants sont mis dans la situation suivante : leur équipe a été mandatée pour conseiller le Ministère des transports afin de faire de ce projet un exemple en matière d'intégration des principes de DD. Ils doivent donc, au moyen de la grille d'analyse, identifier des priorités d'action en matière de DD et proposer des améliorations sur les objectifs pour lesquels le projet démontre des faiblesses.

Les résultats obtenus lors de ces deux exercices n'ont pas fait l'objet d'une recherche formelle. Toutefois, une analyse qualitative a été faite pour y repérer des éléments qui s'inscrivent dans des finalités, des approches ou des stratégies autres que celles qui dominent dans les formations en génie.

Bien entendu, plusieurs des solutions proposées sont des solutions qui s'ancrent dans une finalité technocentrée du développement durable, qui font appel à des approches technoscientifiques ou économicistes, ainsi qu'à des stratégies descendantes, prescriptive, pragmatique, autoritaire et prévisionnelles. Ces approches et stratégies sont répandues dans les pratiques d'ingénierie. Notons par exemple les quelques exemples suivants :

- L'intégration obligatoire de pistes cyclables le long du parcours.

- Les compensations financières offertes aux résidents qui subiront des nuisances associées au nouveau projet.

- L'innovation technologique comme l'utilisation de matériaux écologiques ou le recyclage du bitume pour la construction de la nouvelle route.

- L'utilisation de technique de pointe pour la protection des eaux à proximité de la route.

Toutefois, la présentation préalable de la typologie du DD a servi de prétexte pour inviter les ingénieurs en formation à proposer des solutions innovantes, qui ne sont pas nécessairement des propositions techniques. C'est ainsi que plusieurs des bonifications ont été proposées s'inscrivent dans d'autres finalités, approches et stratégies du DD, comme le démontrent les exemples suivants :

- Créer un comité d'idéation avec les citoyens pour tenter de trouver des solutions aux problématiques rencontrées (approche éthique et stratégie participative).

- Procéder à une identification participative des milieux à préserver pour des raisons écologiques, paysagères ou patrimoniales (approche territoriale et stratégie participative).

- Favoriser le recours à des entreprises régionales pour la construction de la route (stratégie endogène).
- Impliquer des entreprises d'économie sociale qui favorisent la réinsertion dans la réalisation du projet (approche humaniste).

- Jumeler la construction de la nouvelle route au développement d'infrastructures permettant la mise en valeur des attraits touristiques de la région (approche territoriale et stratégie endogène).

- Mettre en place des mécanismes pour réduire la vitesse et augmenter la sécurité aux abords de la nouvelle route (finalité sociocentrée et approche humaniste).

- Mettre en place des mesures de suivi pour connaitre l'impact économique de la voie de contournement sur les commerces locaux (approche planificatrice).

- Profiter du désengorgement futur dans le village pour mettre en valeur les initiatives de transport actif telles la marche et vélo pour favoriser la santé des populations (finalité sociocentrée et approche humaniste).

- Mettre en valeur les services et les attraits touristiques de la municipalité et en faciliter l'accès à partir de la voie de contournement (approche territoriale).

- S'associer au club de marche et au club de motoneige pour développer, à partir de la nouvelle route, des sentiers qui donnent accès à la nature environnante (stratégie globale et participative).

- Créer des stationnements incitatifs pour favoriser le covoiturage (finalité sociocentrée).

- Contourner un milieu humide fragile et aménager, à proximité, une halte routière incluant des panneaux de sensibilisation à la protection de l'environnement (approche environnementaliste).

- Revoir le tracé de la route pour éviter de fractionner une terre agricole (approche territoriale).

- Aménager sous la nouvelle route des passages pour les amphibiens, les reptiles et les petits mammifères (approche environnementaliste).

Ces exemples illustrent comment le DD et les solutions pour une meilleure prise en compte de ces principes peuvent être abordés de multiples façons et en ayant recours à des expertises diverses.

\section{CONCLUSION}

Les solutions pour une meilleure prise en considération des principes de DD dans les projets d'ingénierie passent par une multitude d'approches et de stratégies. Nous ne portons aucun jugement de valeurs sur la pertinence et l'efficacité des actions qui sont habituellement proposées par les ingénieurs. De même, nous n'abordons pas les diverses interprétations du DD dans une approche critique. Les finalités, approches et stratégies les plus fréquemment utilisées par les

CEEA13; 
ingénieurs ont, comme les autres, le potentiel de faire évoluer un projet vers une meilleure intégration du DD.

Toutefois, il importe que les ingénieurs soient conscients que ces multiples interprétations peuvent être portées par les diverses parties prenantes aux projets d'ingénierie. Ainsi, il est selon nous important de sensibiliser les ingénieurs en formation à ces différentes visions du $\mathrm{DD}$, de les préparer à être confrontés à ces multiples interprétations. Les parties prenantes aux projets ont des intérêts qui touchent les dimensions sociale, éthique et de gouvernance du DD, pour lesquelles il n'existe pas toujours de solution technique ou économiques. La formation des ingénieurs doit leur permettre de comprendre ces enjeux et de les intégrer en amont des processus de planification de projet.

C'est à cette fin que la typologie et la grille d'analyse de DD de la chaire en éco-conseil ont été présentées à des étudiants en génie. Les travaux pratiques réalisés par la suite au moyen de ces outils nous ont démontré que leur utilisation dans la formation des ingénieurs facilite l'identification de solutions variées pour répondre aux enjeux sociaux, environnementaux et économiques des projets d'ingénierie, une compétence essentielle pour le travail en équipe multidisciplinaire sur des projets de DD.

\section{Références}

[1] D. Clifton, "Representing A Sustainable World - A Typology Approach“, Journal of Sustainable Development, 3, 2, pp. 40-58, 2010.

[2] A. Dobson, "Environmental Sustainabilities: An Analysis and a Typology“, Environmental Politics, 5, pp. 401-428, 1996.

[3] Christiane Gagnon, "Le développement durable : un nouveau paradigme scientifique? " in PUQ (Ed.), Sciences $d u$ territoire, perspectives québécoises, Massicotte, G. (dir), pp. 335-368, 2008.

[4] P. Jacobs, et B. Saddler, Développement durable et évaluation environnementale : perspectives de planification d'un avenir commun, Hull, Conseil canadien de la recherche sur l'évaluation environnementale, 1990, 204 p.

[5] Annelise Mathieu. et Richard, Soparnot, "Les stratégies de développement durable“, In le développement durable: théories et application au management, Wolff (dir), $2^{\mathrm{e}}$ édition, 2008.

[6] R. Passet, Le développement durable : De la transdisciplinarité à la responsabilité, Congrès de Locarno, 30 avril - 2 mai 1997.

[7] Jean-Pierre Revéret, et Corrine Gendron, "Le développement durable, entre développement et environnement", Liaison Énergie-Francophonie, IEPF, Spécial Sommet de Johannesburg, 55-56-57, p. 33-36, 2002.

[8] Olivier Riffon, et Claude Villeneuve, "Une typologie du développement durable", Liaison Énergie Francophonie, numéro spécial Rio +20, 2011.

[9] Olivier Riffon, et Claude Villeneuve, "Les outils du développement durable, état des lieux", Liaison Énergie Francophonie, numéro spécial Rio +20, 2011.

[10] Léa Sébastien, et Christian Brodhag, "A la recherche de la dimension sociale du développement durable", Développement durable et territoires, Dossier 3 : Les dimensions humaine et sociale du Développement Durable, 2004.

[11] David Tremblay, Najoua Bensalah, Hélène Côté, et Claude Villeneuve, Rapport de la démarche d'analyse de développement durable du projet minier Arnaud selon la grille d'analyse de la Chaire en éco-conseil, Chaire de recherche en éco-conseil, Chicoutimi, 2013.

[12] Suzanne Vallance, and Harvey Perkins, "Is another city possible? Towards an urbanised sustainability", City, 14: 4, pp. 448-456, 2010.

[13] Claude Villeneuve, et Olivier Riffon, Comment réaliser une analyse de développement durable? Grille d'analyse de la Chaire de recherche en éco-conseil. Département des sciences fondamentales, Université du Québec à Chicoutimi, 2011.

[14] Claude Villeneuve, et Olivier Riffon, Comment réaliser une analyse de développement durable? Guide d'utilisation de la grille d'analyse de la Chaire de recherche en écoconseil, Département des sciences fondamentales, Université du Québec à Chicoutimi, 2011. 